\title{
Dislipidemia em pacientes HIV/AIDS em uso de anti-retrovirais num hospital universitário, Rio de Janeiro, Brasil
}

\author{
Dyslipidemia in HIV/AIDS patients in antiretroviral therapy in a university hospital, Rio de Janeiro, Brazil
}

Lidia Farhi'; Dirce Bonfim de Lima²; Cynthia B. Cunha ${ }^{3}$

\begin{abstract}
unitermos
Dislipidemia

AIDS

Anti-retrovirais

\section{resumo}

Introdução: O uso contínuo da terapêutica anti-retroviral (TARV) está associado à ocorrência de diversos distúrbios metabólicos. Objetivo: O principal objetivo deste estudo foi determinar a prevalência de dislipidemia em pacientes com vírus da imunodeficiência humana/síndrome de imunodeficiência adquirida (HIV/AIDS) atendidos no ambulatório de doenças infecciosas do Hospital Universitário Pedro Ernesto da Universidade do Estado do Rio de Janeiro (HUPE/UERJ). Material e métodos: No período de $1 / 10 / 2004$ a $30 / 5 / 2005$, os participantes do estudo responderam a uma entrevista sobre dados demográficos e relativos à saúde. Após a entrevista foram verificadas as seguintes medidas: peso, altura, cintura e quadril. Foi coletado sangue para realização de dosagem plasmática de colesterol total (CT), colesterol da lipoproteína de alta densidade (HDL-C) e triglicerídeos (TG). Dos 268 pacientes que compareceram à consulta no período de estudo, 23 não se apresentaram para a coleta de sangue $\mathrm{e}$ 10 não quiseram participar. O modelo de regressão de Poisson foi empregado para encontrar variáveis associadas à dislipidemia. Resultados: Foram incluídos 235 pacientes, dos quais $182(77,5 \%)$ tinham dislipidemia; houve prevalência maior no sexo masculino $(69,8 \%)$ do que no feminino $(30,2 \%)$; e $26,9 \%$ tinham antecedentes familiares de dislipidemia contra $15,1 \%$ sem este antecedente. Para o tempo de uso de TARV, tanto a média quanto a mediana foram maiores no grupo de pacientes com dislipidemia. Conclusão: Em nosso estudo, a prevalência de dislipidemia em portadores de HIV/AIDS foi alta $(77,5 \%)$ e foram identificados sexo masculino, história familiar de dislipidemia e tempo de uso de TARV como fatores associados.
\end{abstract}

Background: The continuous use of antiretroviral therapy (ART) is associated with several metabolic disturbances. Objectives: The main objective of this study was to determine the prevalence of dyslipidemia in human immunodeficiency virus/acquired immunological deficiency syndrome (HIV/AIDS) patients followed in the infectious diseases outpatient clinic of Hospital Universitário Pedro Ernesto, of Universidade do Estado do Rio de Janeiro (HUPE/UERJ). Material and methods: From 10/1/2004 to 5/30/2005, the participants of this study answered a survey about demographic and health data. Afterwards, the following measurements were checked: weight, height, waist and hips. Blood samples were collected for total cholesterol, high-density lipoprotein cholesterol (HDL-C) and triglycerides tests. Out of 268 patients who came to the appointment during the study period, 23 did not attend the blood collection, and 10 did not want to participate. Poisson regression model was used to find the variables associated with dyslipidemia. Results: Out of the 235 patients included in the study 182 (77.5\%) had dyslipidemia, with prevalence of male (69.8\%) over female ones (30.2\%). Among the patients with dyslipidemia, 26.9\% had family history of dyslipidemia against $15.1 \%$ who did not. Regarding ART duration, both mean and media were higher in the group of patients with dyslipidemia. Conclusion: In our study the prevalence of dyslipidemia in HIV/AIDS patients was high (77.5\%), and the associated factors were male sex, family history of dyslipidemia and ART duration.

key words

Dyslipidemias

AIDS

Antiretroviral agents

1. Bióloga da Faculdade de Ciências Médicas da Universidade do Estado do Rio de Janeiro (UER)).

2. Doutora em Medicina; professora adjunta do Hospital Universitário Pedro Ernesto (HUPE), da UERJ.

3. Mestre em Saúde Coletiva; auxiliar de pesquisa do Instituto de Pesquisa Evandro Chagas (IPEC) da Fundação Oswaldo Cruz, (FIOCRUZ). 


\section{Introdução}

Em 1987, foi aprovada para uso a primeira droga para tratamento da síndrome de imunodeficiência adquirida (AIDS), a zidovudina, inicialmente usada em monoterapia até a liberação da didanosina('). A seguir, novos medicamentos anti-retrovirais (ARV) surgiram, não só inibidores de transcriptase reversa nucleosídeos análogos (ITRN), como não-nucleosídeos (ITRNN) e nucleotídeos, inibidores de protease (IP), inibidores de fusão (IF) e, mais recentemente, inibidores de integrase (II) e de receptores de CCR5.

A partir de 1996, com a introdução da terapêutica antiretroviral (TARV) de alta potência, observou-se redução na freqüência das doenças oportunistas, assim como diminuição do número de mortes atribuídas à AIDS(32).

Com o uso da TARV observamos melhora da qualidade de vida e aumento da sobrevida, mas também encontramos distúrbios metabólicos caracterizados por hiperglicemia, dislipidemia e alterações corporais (lipodistrofia).

O objetivo de nossa pesquisa foi estudar a prevalência de dislipidemia em um grupo de pacientes com AIDS em uso de TARV e avaliar os possíveis fatores relacionados.

\section{Material e métodos}

\section{Desenho do estudo}

Trata-se de um estudo de corte transversal para avaliação da prevalência de dislipidemia em pacientes adultos, de ambos os sexos, com vírus da imunodeficiência humana (HIV)/AIDS, acompanhados em serviço ambulatorial de infectologia do Hospital Universitário Pedro Ernesto, da Universidade do Estado do Rio de Janeiro (HUPE/UERJ), entre $1^{\circ}$ de outubro de 2004 e 30 de maio de 2005.

\section{Coleta de dados}

Utilizamos questionário contendo dados demográficos, história patológica pregressa, hábitos pessoais, história familiar e medidas antropométricas.

\section{Etilismo}

Foram considerados não-etilistas os indivíduos que nunca ingeriram álcool na vida; etilistas moderados, homens que ingeriam até 21 doses de bebidas alcoólicas por semana e mulheres que ingeriam até 14 doses por semana; etilistas pesados, os que ingeriam quantidades superiores a essas semanalmente ${ }^{(20)}$.

\section{Tabagismo}

Foi considerado tabagista o paciente que era fumante no momento da entrevista.

\section{Atividades físicas}

Foram considerados dois grupos: os de vida sedentária e os que praticavam pelo menos alguma atividade física, como musculação, aeróbica, caminhada, pedalada ou corrida.

\section{Revisão de prontuário médico}

Verificamos nos registros médicos: sorologia para hepatites $\mathrm{B} \mathrm{eC}$, subtipagem de linfócitos (exame imediatamente anterior à entrevista) e quantificação do ácido ribonucleico (RNA) do HIV (exame imediatamente anterior à entrevista), dados sobre o uso da TARV (todos os esquemas utilizados, incluindo nome das drogas e tempo de uso de cada uma), a confirmação do diagnóstico de hipertensão arterial e de diabetes.

\section{Exames laboratoriais}

Após 12 horas de jejum foram coletados $10 \mathrm{ml}$ de sangue de cada participante, em tubos contendo gel. As dosagens de colesterol total (CT), colesterol da lipoproteína de alta densidade (HDL-C) e triglicerídeos (TG) foram obtidas a partir da utilização de kits da Roche, por método enzimático automatizado e leitura no aparelho Roche Modular. O cálculo para a dosagem de colesterol da lipoproteína de baixa densidade (LDL-C) baseou-se na seguinte fórmula de Friedwald: $\mathrm{LDL}-\mathrm{C}=\mathrm{CT}-\mathrm{HDL}-\mathrm{C}-\mathrm{TG} / 5^{(15)}$.

\section{Conceito de dislipidemia}

Alteração nas dosagens séricas de CT, HDL-C, LDL-C e/ou TG, ou seja, CT ou TG acima do valor normal ( $\geq 200$ $\mathrm{mg} \%$ e $\geq 150 \mathrm{mg} \%$, respectivamente), qualquer dosagem de HDL-C abaixo do valor normal ( $\leq 40 \mathrm{mg} \%)$ e LDL-C $\geq$ $130 \mathrm{mg} / \mathrm{dl}^{(15)}$.

\section{Medidas antropométricas}

$\mathrm{Na}$ ocasião da entrevista foram verificados os seguintes dados antropométricos:

- peso e altura - vestidos e descalços, em balança tipo Filizola;

- medida de cintura e quadril - com fita métrica, na linha umbilical e no nível da crista ilíaca;

- cálculo do índice de massa corporal (IMC) - IMC = peso $(\mathrm{kg}) /$ altura $(\mathrm{m})^{(2)}$. Foram considerados com sobrepeso os pacientes com IMC $\geq 25$; 
- cálculo da relação cintura/quadril (RCQ), em centímetros - relações maiores que 0,9-1 para homens e 0,8 para muIheres foram consideradas fator de risco cardíaco ${ }^{(16)}$.

\section{Avaliação imunológica e carga viral}

Foram registrados os resultados de contagem de células CD4 disponíveis durante o período de acompanhamento: - nadir de CD4 - encontrado a partir da procura do menor valor de CD4 observado ao longo do acompanhamento;

- último CD4 - último valor observado a partir de uma janela anterior a seis meses da data da coleta;

- maior valor de carga viral - encontrado a partir da procura do maior valor observado de carga viral ao longo do acompanhamento do paciente;

- última carga viral - último valor observado a partir de uma janela anterior a seis meses da data da coleta.

\section{Análise estatística}

Foram realizadas análises univariadas com o emprego dos testes $\chi^{2}$ de Pearson e exato de Fisher para as variáveis categóricas e, para as variáveis contínuas, de análise de variância e do teste não-paramétrico de Kruskal-Wallis.

Para a análise multivariada, a seleção das variáveis foi realizada com base nos dados conhecidos da literatura, na plausibilidade biológica e na significância estatística $(p \leq 0,25)$ encontrada na análise univariada. Considerou-se co-variável fixa no modelo o tempo de uso de medicamentos anti-retrovirais.

No modelo multivariado, todas as variáveis selecionadas foram testadas e, no modelo final, permaneceram as variáveis estatisticamente significativas em $5 \%(p \leq 0,05)$, algumas com significância limítrofe $(p \leq 0,1)$, bem como outras que apresentaram efeito considerado importante (acima de 30\% para proteção ou risco). Empregou-se o modelo de regressão de Poisson com variância robusta para a estimação da razão de prevalência devido à elevada prevalência de dislipidemia encontrada.

Os dados foram compilados usando-se o programa Epi Info, versão 6.04. Os programas utilizados para a análise estatística foram o R 2.5.1 para a análise univariada e o Stata 10 para os modelos de regressão de Poisson com variância robusta.

\section{Aspectos éticos}

O protocolo de pesquisa foi avaliado e aprovado pelo Comitê de Ética em Pesquisa do HUPE. Nenhum procedimento do estudo foi realizado antes que cada voluntário assinasse o Termo de Consentimento Livre e Esclarecido (TCLE).

\section{Resultados}

\section{População em estudo}

A população consistiu de 268 pacientes, entre os quais 10 se recusaram a participar do estudo e 23 não compareceram para a coleta de sangue.

\section{Prevalência de dislipidemia}

A dislipidemia foi prevalente em 77,5\% (182 pacientes). Cinqüenta e três pacientes (22,5\%) possuíam valores normais de TG, CT, HDL-C e LDL-C. A prevalência das alterações no valor sérico de cada um dos lípides avaliados é descrita na Tabela 1.

\section{Dados demográficos}

As características demográficas dos 235 pacientes estão apresentadas na Tabela 2. Observa-se que o sexo masculino apresentou maior prevalência de dislipidemia do que o feminino, com $69,8 \%$ e $30,2 \%$, respectivamente $(p<0,05)$.

A média de idade foi de 42,8 anos entre os pacientes com dislipidemia e 41,2 anos entre os sem dislipidemia $(p>0,05)$. Quanto à etnia, observou-se que a distribuição dos não-brancos nos grupos sem e com dislipidemia ocorreu com pequena diferença, com percentuais de $58,5 \%$ para os que não apresentaram dislipidemia e $54,9 \%$ para os que a apresentaram $(p>0,05)$.

Características clinicolaboratoriais relativas aos níveis plasmáticos de CT, HDL-C, LDL-C e TG de pacientes HIV-positivos em vigência de

Tabela 1 tratamento anti-retroviral

\begin{tabular}{lc}
\hline Parâmetros & $n(\%)$ \\
CT $\geq 200(n=235)$ & $67(28,5)$ \\
LDL-C $\geq 130(n=232)^{*}$ & $42(18,1)$ \\
HDL-C $\leq 40(n=235)$ & $137(58,3)$ \\
TG $\geq 150(n=235)$ & $105(44,7)$ \\
TG $>500(n=235)$ & $14(6)$ \\
LDL-C $\geq 190(n=232)^{*}$ & $4(1,7)$ \\
CT $\geq 240(n=235)$ & $28(11,9)$ \\
TG $\geq 150$ e colesterol $\geq 200(n=235)$ & $48(20,4)$ \\
\hline
\end{tabular}

*Três pacientes apresentaram triglicerídeos $>400 \mathrm{mg} / \mathrm{dl}$.

CT: colesterol total ; LDL-C: colesterol da lipoproteína de baixa densidade; HDL-C: colesterol da lipoproteína de alta densidade ; TC: triglicerídeos. 


\begin{tabular}{|c|c|c|c|c|}
\hline \multirow{2}{*}{$\begin{array}{l}\text { Tabela } 2 \\
\text { Variáveis }\end{array}$} & \multicolumn{4}{|c|}{$\begin{array}{l}\text { Características sociodemográficas de pacientes HIV-positivos em vigência de tratamento } \\
\text { anti-retroviral }\end{array}$} \\
\hline & Pacientes sem dislipidemia & Pacientes com dislipidemia & Total & $p$-valor \\
\hline \multicolumn{5}{|l|}{ Sexo } \\
\hline$n(\%)$ & & & & 0,018 \\
\hline Feminino & $26(49,1)$ & $55(30,2)$ & $81(34,5)$ & \\
\hline Masculino & $27(50,9)$ & $127(69,8)$ & $154(65,5)$ & \\
\hline Idade (anos) & & & & 0,294 \\
\hline Média ( \pm EP) & $41,2(0,6)$ & $42,8(0,6)$ & $42,4(0,6)$ & \\
\hline Mediana (IQR) & $39,5(37,1-48,5)$ & $42,1(34,1-47)$ & $42(36,5-48,3)$ & \\
\hline \multicolumn{5}{|l|}{ Raça } \\
\hline$n(\%)$ & & & & 0,764 \\
\hline Não-branco & $31(58,5)$ & $100(54,9)$ & $131(55,7)$ & \\
\hline Branco & $22(41,5)$ & $82(45,1)$ & $104(44,3)$ & \\
\hline Total & $53(100)$ & $182(100)$ & $235(100)$ & \\
\hline
\end{tabular}

EP: erro padrão; IQR: intervalo interquartil.

\section{História e hábitos pessoais}

- Tempo de acompanhamento dos pacientes: média de 62,2 meses (erro padrão $[E P] \pm 2,6$ ), com dislipidemia 65 meses $(E P \pm 2,6)$ e 53,8 (EP $\pm 2,8)$ sem dislipidemia $(p>0,05)$;

- revisão dos prontuários: $15,7 \%$ e 5,1\% dos pacientes tinham história de hipertensão e diabetes, respectivamente, $17 \%$ e $5,7 \%$ sem dislipidemia e 15,4\% e 4,9\% com dislipidemia ( $p>0,05$ em ambos);

- marcadores virais: 3,3\% com marcador positivo para vírus B e dislipidemia, os sem dislipidemia não apresentavam marcador positivo. Co-infecção com vírus C: 7,5\% sem dislipidemia e 6,6\% com dislipidemia ( $p>0,05$ em ambos);

- tabagismo: 28,3\% dos tabagistas não apresentaram dislipidemia, enquanto $31,3 \%$ a apresentaram $(p>0,05)$;

- etilismo: entre os que não apresentaram dislipidemia, os que nunca fizeram uso de bebida alcoólica, bebiam moderadamente e bebiam pesado corresponderam a, respectivamente, $67,9 \%$, 24,5\% e 7,5\%, enquanto nos dislipidêmicos a equivalência foi de 73,1\%, 21,4\% e 5,5\% ( $p>0,05)$;

- prática de atividades físicas: entre os dislipidêmicos correspondeu a $29,7 \%$ contra $15,1 \%$ nos pacientes sem dislipidemia $(p>0,05)$ (Tabela 3).

\section{Medidas antropométricas}

A ocorrência de IMC $\geq 25$ foi de 32,1\% no grupo sem dislipidemia e $41,8 \%$ no com dislipidemia $(p>0,05)$. Relação cintura-quadril (RCQ) aumentada: 35,8\% sem dislipidemia contra 26,9\% com dislipidemia ( $p>0,05)$ (Tabela 4).

\section{Antecedentes familiares}

Entre os pacientes com dislipidemia, 26,9\% tinham antecedentes familiares de dislipidemia e 52,2\%, de coronariopatia. Esses antecedentes familiares foram referidos por $15,1 \%$ e $56,6 \%$ dos pacientes sem dislipidemia $(p>0,05)$ (Tabela 5).

\section{Esquemas anti-retrovirais}

Para tempo de exposição aos ITRNs, tempo de exposição a análogos timidínicos (AZT/D4T), número de ARVs usados, número de esquemas ARV usados e tempo de TARV, tanto a média quanto a mediana foram maiores no grupo de pacientes com dislipidemia. Em todas essas variáveis houve pelo menos uma diferença estatisticamente significativa entre os grupos $(p<0,05)$.

Para as variáveis tempo de exposição aos análogos timidínicos (AZT/D4T) e tempo de exposição aos ITRNs não se verificou a existência de diferença significativa entre os grupos quanto à comparação de proporções $(p>0,05)$.

A prevalência de uso de IP foi maior nos pacientes que apresentaram dislipidemia, com 53,5\%, e com 34,7\% naqueles que não a apresentaram $(p<0,05)$.

Não houve diferença estatisticamente significativa para tempo de exposição aos ITRNNs $(p>0,05)$ (Tabela 6). 
Tabela 3 História e hábitos pessoais de pacientes HIV-positivos em vigência de tratamento anti-retroviral

\begin{tabular}{|c|c|c|c|c|}
\hline Variáveis & $\begin{array}{c}\text { Pacientes sem } \\
\text { dislipidemia } \\
n(\%)\end{array}$ & $\begin{array}{c}\text { Pacientes com } \\
\text { dislipidemia } \\
n(\%)\end{array}$ & $\begin{array}{c}\text { Total } \\
n(\%)\end{array}$ & $p$-valor \\
\hline Hipertensão arterial & & & & 1 \\
\hline Não & $44(83)$ & $154(84,6)$ & $198(84,3)$ & \\
\hline Sim & $9(17)$ & $28(15,4)$ & $37(15,7)$ & \\
\hline Diabetes & & & & 0,735 \\
\hline Não & $50(94,3)$ & $173(95,1)$ & $223(94,9)$ & \\
\hline Sim & $3(5,7)$ & $9(4,9)$ & $12(5,1)$ & \\
\hline Hepatite B & & & & 0,342 \\
\hline Não & $53(100)$ & $176(96,7)$ & $229(97,4)$ & \\
\hline Sim & - & $6(3,3)$ & $6(2,6)$ & \\
\hline \multicolumn{5}{|l|}{ Hepatite C } \\
\hline Não & $49(92,5)$ & $170(93,4)$ & $219(93,2)$ & \\
\hline $\operatorname{Sim}$ & $4(7,5)$ & $12(6,6)$ & $16(6,8)$ & \\
\hline Tabagismo & & & & 0,803 \\
\hline Não & $38(71,7)$ & $125(68,7)$ & $163(69,4)$ & \\
\hline $\operatorname{Sim}$ & $15(28,3)$ & $57(31,3)$ & $72(30,6)$ & \\
\hline Etilismo & & & & 0,65 \\
\hline Bebe moderado & $13(24,5)$ & $39(21,4)$ & $52(22,1)$ & \\
\hline Bebe pesado & $4(7,5)$ & $10(5,5)$ & $14(6)$ & \\
\hline Não bebe & $36(67,9)$ & $133(73,1)$ & $169(71,9)$ & \\
\hline Prática de atividades físicas & & & & 0,052 \\
\hline Não & $45(84,9)$ & $128(70,3)$ & $173(73,6)$ & \\
\hline Sim & $8(15,1)$ & $54(29,7)$ & $62(26,4)$ & \\
\hline Total & $53(100)$ & $182(100)$ & $235(100)$ & \\
\hline
\end{tabular}

Medidas antropométricas e antecedentes familiares de pacientes HIV-positivos em vigência de Tabela 4 tratamento anti-retroviral

\begin{tabular}{lcccc}
\hline Variáveis & $\begin{array}{c}\text { Pacientes sem dislipidemia } \\
n(\%)\end{array}$ & $\begin{array}{c}\text { Pacientes com dislipidemia } \\
n(\%)\end{array}$ & $\begin{array}{c}\text { Total } \\
n(\%)\end{array}$ & $p$-valor \\
Índice de massa corporal & & & & 0,267 \\
$<25$ & $36(67,9)$ & $106(58,2)$ & $142(60,4)$ & \\
$\geq 25$ & $17(32,1)$ & $76(41,8)$ & $93(39,6)$ & 0,276 \\
RCQ* & & & & \\
RCQ não aumentada & $34(64,2)$ & $133(73,1)$ & $167(71,1)$ & \\
RCQ aumentada & $19(35,8)$ & $49(26,9)$ & $68(28,9)$ & \\
Total & $53(100)$ & $182(100)$ & $235(100)$ & \\
\hline
\end{tabular}

*Os valores da RCQ foram calculados de acordo com os padrões ${ }^{(16)}$ para cada sexo (homens: $\geq 0,9$ e $\leq 1$; mulheres: $\geq 0,8$ ); RCQ: relação cintura quadril.

\section{Categoria de exposição ao HIV}

As exposições heterossexual, entre homens que fazem sexo com homens ( $\mathrm{HSH}$ ) e bissexual foram, respectivamente, $62,3 \%, 18,9 \%$ e $7,5 \%$ no grupo sem dislipidemia e $51,1 \%$,
$29 \%$ e 5,1\% naquele com dislipidemia. Transfusão de sangue e uso de drogas injetáveis (UDI) foram citadas por 7,5\% e 1,9\% dos indivíduos sem dislipidemia e 4,9\% e 3,3\% do grupo com dislipidemia, respectivamente $(p>0,05)$ (Tabela 7). 


\section{Tabela 5 Antecedentes familiares de pacientes HIV-positivos em vigência de tratamento anti-retroviral}

\begin{tabular}{|c|c|c|c|c|}
\hline Variáveis & $\begin{array}{l}\text { Pacientes sem dislipidemia } \\
\qquad n(\%)\end{array}$ & $\begin{array}{l}\text { Pacientes com dislipidemia } \\
\qquad n(\%)\end{array}$ & $\begin{array}{l}\text { Total } \\
n(\%)\end{array}$ & $p$-valor \\
\hline $\begin{array}{l}\text { Histórico familiar de } \\
\text { dislipidemia }\end{array}$ & & & & 0,113 \\
\hline Não & $45(84,9)$ & $133(73,1)$ & $178(75,7)$ & \\
\hline Sim & $8(15,1)$ & $49(26,9)$ & $57(24,3)$ & \\
\hline $\begin{array}{l}\text { Histórico familiar de } \\
\text { coronariopatia }\end{array}$ & & & & 0,682 \\
\hline Não & $23(43,4)$ & $87(47,8)$ & $110(46,8)$ & \\
\hline Sim & $30(56,6)$ & $95(52,2)$ & $125(53,2)$ & \\
\hline Total & $53(100)$ & $182(100)$ & $235(100)$ & \\
\hline
\end{tabular}

\section{Tabela 6 Esquemas de classes anti-retrovirais de pacientes HIV-positivos em vigência de tratamento anti- retroviral}

Variáveis

$n(\%)$

Pacientes sem dislipidemia Pacientes com dislipidemia

Total

$$
(n=53) \quad(n=182) \quad(n=235)
$$

Média em meses ( \pm EP)

$$
49 \text { (100) }
$$

$157(100)$

206 (100)

0,342

Mediana em meses (IQR)

$$
53(2,8)
$$

$65,8(2,5)$

$62,8(2,6)$

0,051

Tempo de exposição a IP (meses)

$n(\%)$

$47,1(37,1-87,5)$

$67,2(16-78,2)$

$$
17(34,7)
$$

$53(3,3)$

Mediana em meses (IQR)

$$
62,6(29,6-74,1)
$$

$$
84(53,5)
$$$$
52,1(2,9)
$$$$
57,3(14,2-81)
$$

Tempo de exposição aos NNRTIs (meses)

$n(\%)$

Média em meses ( \pm EP)

Mediana em meses (IQR)
$39(79,6)$

$30,1(1,6)$

$$
121(77,1)
$$

$32,4(1,6)$

\begin{tabular}{|c|c|c|c|c|}
\hline$n(\%)$ & $49(100)$ & $154(98,1)$ & $203(98,5)$ & 0,176 \\
\hline Média em meses ( $\pm E P$ ) & $50,4(2,5)$ & $64,1(2,4)$ & $60,8(2,4)$ & 0,021 \\
\hline Mediana em meses (IQR) & $47,1(37,9-85,9)$ & $65,6(16,0-78,2)$ & $61,4(33,8-85,8)$ & 0,023 \\
\hline \multicolumn{5}{|l|}{ Número de ARVs usados } \\
\hline Média $( \pm E P)$ & $4,3(0,1)$ & $5,3(0,2)$ & $5,1(0,2)$ & 0,006 \\
\hline Mediana (IQR) & $3(3-7)$ & $5(3-5)$ & $4(3-6)$ & 0,006 \\
\hline \multicolumn{5}{|c|}{ Número de esquemas ARV usados } \\
\hline Média $( \pm E P)$ & $2,4(0,1)$ & $3,3(0,2)$ & $3,1(0,2)$ & 0,014 \\
\hline Mediana (IQR) & $2(1-4)$ & $3(1-3)$ & $2(1-4)$ & 0,022 \\
\hline \multicolumn{5}{|l|}{ Tempo de TARV (meses) } \\
\hline Média em meses ( \pm EP) & $53(2,8)$ & $66,4(2,6)$ & $63,2(2,7)$ & 0,042 \\
\hline Mediana em meses (IQR) & $47,1(37,1-89,5)$ & $67,2(16-78,2)$ & $61,8(33,7-87,5)$ & 0,023 \\
\hline
\end{tabular}

$29,7(14,5-49,8)$

$31,9(13,9-46,4)$

$62,8(2,6)$

0,026

p-valor

Tempo de exposição aos análogos timidínicos (AZT/D4T) (meses)

ITRN: inibidores de transcriptase reversa nucleosídeos; IP: inibidores de protease; EP: erro padrão; IQR: intervalo interquartil; ITRNN: inibidores de transcriptase reversa nãonucleosídeos; ARV: anti-retrovirais; TARV: terapêutica anti-retroviral. 


\section{Tabela 7 Categoria de exposição de pacientes HIV-positivos em vigência de tratamento anti-retroviral}

\begin{tabular}{lcccc}
\hline Variáveis & Pacientes sem dislipidemia & Pacientes com dislipidemia & Total & \\
& $n(\%)$ & $n(\%)$ & & $p$-valor \\
Categoria de exposição & & & & 0,215 \\
Bissexual & $4(7,5)$ & $9(4,9)$ & $13(5,5)$ & \\
Heterossexual & $33(62,3)$ & $90(49,5)$ & $123(52,3)$ & \\
HSH & $10(18,9)$ & $52(28,6)$ & $62(26,4)$ & $13(5,5)$ \\
Transfusão & $4(7,5)$ & $9(4,9)$ & $7(3)$ \\
UDI & $1(1,9)$ & $6(3,3)$ & $17(7,2)$ \\
Ignorada & $1(1,9)$ & $16(8,8)$ & $235(100)$ \\
Total & $53(100)$ & $182(100)$ & \\
\hline
\end{tabular}

HSH: homens que fazem sexo com homens; UDI: uso de drogas injetáveis.

\section{Avaliação imunológica e carga viral}

Para o nadir de CD4, tanto a média quanto a mediana foram menores no grupo com dislipidemia quando comparados ao grupo sem dislipidemia, enquanto para o último valor de CD4 ocorreu o contrário. Não houve diferença estatisticamente significativa entre os grupos para as duas variáveis $(p>0,05)$.

A mediana do último valor de carga viral foi igual nos grupos com e sem dislipidemia, enquanto a média e a mediana do maior valor de carga viral foram maiores com dislipidemia $(p>0,05)$ (Tabela 8).

\section{Modelo multivariado}

A Tabela 9 apresenta as estimativas da regressão de Poisson para as variáveis que permaneceram no modelo. Em relação ao tempo de uso de ARV observou-se uma razão de prevalência (RP) estimada de 1,143 (intervalo de confiança [IC] 95\% 1,009-1,294) para um aumento de 60 meses de acompanhamento.

A RP para os pacientes do sexo masculino foi de 1,201 (IC 95\% 1,001-1,44), tendo como categoria de referência o sexo feminino, enquanto os pacientes com histórico familiar de dislipidemia apresentaram RP de 1,178 (IC 95\%

\section{Avaliação imunológica de pacientes HIV-positivos em vigência de tratamento anti-retroviral}

\section{Tabela 8}

\begin{tabular}{|c|c|c|c|c|}
\hline Variáveis* & Pacientes sem dislipidemia & Pacientes com dislipidemia & Total & $p$-valor \\
\hline \multicolumn{5}{|l|}{ Nadir de CD4 } \\
\hline Média ( \pm EP) & $243,8(11,3)$ & $218,5(12,6)$ & $224,1(12,3)$ & 0,366 \\
\hline Mediana (IQR) & $216(93-287,5)$ & $175,5(152,5-291,8)$ & $186,5(103,3-289,3)$ & \\
\hline \multicolumn{5}{|l|}{ Último CD4 } \\
\hline Média ( \pm EP) & $459,5(25,1)$ & $473,8(23,6)$ & $470,4(23,9)$ & 0,808 \\
\hline Mediana (IQR) & $373(320-577)$ & $440(253,5-646,5)$ & $432,5(317,8-587,5)$ & \\
\hline \multicolumn{5}{|c|}{ Maior valor da carga viral ${ }^{* *}$} \\
\hline Média (IC 95\%) & $\begin{array}{c}14.507 \\
(10.190,9-20.651)\end{array}$ & $\begin{array}{c}23.791,1 \\
(16.491,9-34.320,7)\end{array}$ & $\begin{array}{c}21.314,4 \\
(14.815,1-30.664,8)\end{array}$ & 0,261 \\
\hline Mediana (IQR) & $\begin{array}{c}35.496,5 \\
(3.634,4-214.819,9)\end{array}$ & $\begin{array}{c}60.000 \\
(2.565,2-97.767,4)\end{array}$ & $\begin{array}{c}55.000 \\
(3.206,8-167.442,9)\end{array}$ & 0,162 \\
\hline \multicolumn{5}{|c|}{ Última carga viral ${ }^{* * *}$} \\
\hline Média (IC 95\%) & $526,2(334,3-828,4)$ & $526,5(338,6-818,5)$ & $526,4(338,2-819,4)$ & 0,999 \\
\hline Mediana (IQR) & $79(79-3.500)$ & $79(79-4.695,7)$ & $79(79-3.824,8)$ & 0,877 \\
\hline
\end{tabular}




\begin{tabular}{|c|c|c|}
\hline $\begin{array}{l}\text { Razões de prevalência estimadas pelo modelo de rę } \\
\text { pacientes HIV-positivos em vigência de tratamento a }\end{array}$ & $\begin{array}{l}\text { s̃o multivariada de Poi } \\
\text { retroviral }\end{array}$ & ra os \\
\hline Variáveis & RP (IC 95\%) & p-valor \\
\hline $\begin{array}{l}\text { Tempo de uso de ARV em meses (RP estimada para um aumento de } \\
60 \text { meses no tempo de uso de ARV) }\end{array}$ & $1,143(1,009-1,294)$ & 0,035 \\
\hline Sexo & & 0,049 \\
\hline Masculino & $1,201(1,001-1,44)$ & \\
\hline Feminino & 1 & \\
\hline Histórico familiar de dislipidemia & & 0,026 \\
\hline Sim & $1,178(1,019-1,362)$ & \\
\hline Não & 1 & \\
\hline
\end{tabular}

ARV: anti-retrovirais; RP: razão de prevalência.

1,019-1,362) quando comparados aos que não possuíam histórico familiar.

\section{Discussão}

A prevalência de dislipidemia de 77,5\% (182 pacientes) é similar à encontrada por outros autores ${ }^{(9,12,22,27,29)}$. O percentual de hipertrigliceridemia de 44,7 é também

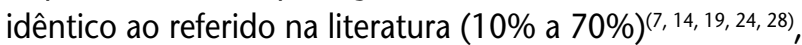
bem como o de hipercolesterolemia ${ }^{(7,27,28)}$.

A alta prevalência de dislipidemia em portadores de HIV é um fato alarmante, pois esse distúrbio está associado ao aumento de doenças cardiovasculares ${ }^{(1,3,6,18,31)}$.

A média de idade dos pacientes envolvidos nesta pesquisa é representativa dos casos de AIDS notificados no Brasil(23) e semelhante à observada em outros estudos sobre prevalência de dislipidemia ${ }^{(7,12,18,27)}$. Não encontramos correlação entre idade e ocorrência de alterações lipídicas, ao contrário do observado por outros autores ${ }^{(2,7)}$. Em nossa casuística, os $65,5 \%$ dos pacientes do sexo masculino apresentaram maior razão de prevalência para dislipidemia do que os do sexo feminino, o que está de acordo com as observações de Araújo et al. ${ }^{(2)}$ e Castro-Sansores et al. ${ }^{(11)}$. Outros autores não confirmaram esses dados $(7,8,12,25,27)$.

A raça branca foi preponderante em nosso grupo (45\%), corroborando os dados da Fundação Instituto Brasileiro de Geografia e Estatística (IBGE)(26) e não foi um fator associado à ocorrência de dislipidemia, ao contrário do referido por outros autores $(4,17,27)$.

Entre antecedentes pessoais, familiares e co-morbidades, apenas a história familiar de dislipidemia permaneceu como variável associada à ocorrência do desfecho primário, observando-se RP de dislipidemia de 1,178 (IC 95\% 1,019-1,362) nos pacientes que relatavam antecedente familiar do distúrbio quando comparados aos que não possuíam esse histórico.
Em relação a outros fatores de risco para eventos cardiovasculares, observamos que a freqüência de tabagismo, etilismo pesado, sedentarismo e o histórico familiar de coronariopatia, diabetes e hipertensão arterial não foram associados à ocorrência de dislipidemia, mas o acúmulo desses fatores aumenta o risco de eventos cardíacos. Outros estudos em portadores do HIV também não associaram dislipidemia ao tabagismo ${ }^{(7,27,29)}$, mas ao antecedente familiar de doença coronariana ${ }^{(27)}$.

Quanto ao sedentarismo, diversos autores observaram que a prática de atividades físicas, especialmente aeróbica, associa-se a valores elevados de HDL e diminuição dos níveis de TG e CT em portadores do HIV(21, 29).

Ao contrário de Bedimo et al. ${ }^{(5)}$, não encontramos correlação entre infecção por vírus $C$ e alterações lipídicas, possivelmente devido à baixa prevalência da infecção em nossa casuística.

A freqüência de pacientes com IMC $\geq 25$ foi de $39,6 \%$, e a de pacientes com RCQ aumentada foi de $28,9 \%$. Apesar de essas alterações não serem fatores relacionados com o desenvolvimento de dislipidemia, sua coexistência está associada, na literatura, ao maior risco cardíaco ${ }^{(13,33)}$.

A dislipidemia foi associada inicialmente ao uso de $\operatorname{IP}^{(22,30)} \mathrm{e}$, mais recentemente, também ao de ITRNs ${ }^{(10,19)}$. Em nosso estudo a prevalência de uso de IP foi maior nos pacientes que apresentaram dislipidemia do que naqueles que não a apresentaram (53,5\% e $34,7 \%$, respectivamente), porém a exposição aos IPs não se manteve como fator associado à dislipidemia no modelo multivariado. Isso pode ser explicado pelo fato de os pacientes em nossa casuística terem usado, em média, cinco medicamentos diferentes, portanto, com exposição a todas as classes.

O tempo de exposição aos ARVs é associado, na literatura, ao risco de desenvolvimento de dislipidemia ${ }^{(7)}$. No nosso estudo, o tempo médio de uso de ARV foi maior 
nos pacientes com dislipidemia que naqueles sem dislipidemia (66,4 meses e 53 meses, respectivamente), variável que se associou positivamente à dislipidemia no modelo multivariado.

Quanto ao número de células de CD4, observamos que não houve diferença estatisticamente significativa entre o grupo de pacientes dislipidêmicos e o de não-dislipidêmicos, ao contrário do relatado por Bonfanti et al. ${ }^{(7)}$.

A dosagem semestral de lípides deve ser feita antes do início da TARV e a cada dois meses após a instituição do tratamento ou após qualquer mudança do esquema ${ }^{(19)}$. Além disso, seria importante a investigação da presença de história familiar para dislipidemia e/ou diabetes, bem como dos hábitos de vida do paciente.

\section{Conclusões}

- Os pacientes do sexo masculino apresentaram maior prevalência de dislipidemia quando comparados aos do sexo feminino;

- história familiar de dislipidemia relacionou-se diretamente com ocorrência de dislipidemia;

- o tempo de uso de ARV associou-se positivamente à dislipidemia;

- o uso da TARV é indispensável por aumentar a expectativa de vida dos pacientes HIV-positivos e diminuir a morbidade e a mortalidade desses pacientes. Faz-se necessário o acompanhamento laboratorial freqüente e a mudança de hábitos de vida para amenizar os possíveis efeitos colaterais associados às classes dos ARVs em uso.

\section{Referências}

1. ACHUTT, A. Epidemiologia das doenças cardiovasculares (DCV): dados do Brasil. Curso de Reciclagem em Cardiologia da Sociedade de Cardiologia do RS/SBC, 2003. Rio Grande do Sul: Sociedade Brasileira de Cardiologia; 2003. Disponível em: http://www.cardiol. com.br.

2. ARAÚJO, P. S. R. et al. Antiretroviral treatment for HIV infection/AIDS and the risk of developing hyperglycemia and hyperlipidemia. Rev Inst Med Trop Sao Paulo, v. 49, n. 2, p. 73-8, 2007.

3. BARBARO, G. et al. Incidence of coronary artery disease in HIV-infected patients receiving or not protease inhibitors: a randomized, multicenter study. In: XIV INTERNATIONAL AIDS CONFERENCE. 2002, Barcelona. Abstract $n^{\circ}$ WeOrB1307.

4. BAUSSERMAN, L. L. et al. Racial differences in serum lipids in HIV+ women treated with protease inhibitor regimens. HIV Clin Trials, v. 5, n. 6, p. 399-405, 2004.

5. BEDIMO, R. et al. Lipid abnormalities in HIV/hepatitis C viruscoinfected patients. HIV Med, v. 7, n. 8, p. 530-6, 2006.

6. BOLLENS, D. et al. Family history, immuno-infectious status and type of antiretroviral drug regimen are predictors for major hypertriglyceridemia in HIV-infected patients. In: 41st INTERSCIENCE CONFERENCE ON ANTIMICROBIAL AGENTS AND CHEMOTERAPY. 2001, Chicago. Abstract nº-220.

7. BONFANTI, P. et al.; CISAI Study Group. Metabolic syndrome: a real threat for HIV-positive patients? Results from the SIMONE study. J Acquir Immune Defic Syndr, v. 42, n. 1, p. 128-31, 2006.

8. BOULASSEL, M. R. et al. Gender and long-term metabolic toxicities from antiretroviral therapy in HIV-1 infected persons. J Med Virol, v. 78, n. 9, p. 1158-63, 2006.

9. BROWN, D. M. et al. Factors affecting attrition in a longitudinal study of patients with AIDS. AIDS Care, v. 18, n. 7, p. 821-9, 2006.
10. CARR, A. Cardiovascular risk factors in HIV-infected patients. J Acquir Immune Defic Syndr, v. 34, Suppl 1, p. S73-8, 2003.

11. CASTRO-SANSORES, C. J. et al. Hyperlipidemia and glucose intolerance in patients with HIV infection receiving antiretroviral therapy. Salud Publica Mex, v. 48, n. 3, p. 193-9, 2006.

12. CHUAPAI, Y. et al. Lipodystrophy and dyslipidemia in human immunodeficiency virus-infected Thai patients receiving antiretroviral therapy. J Med Assoc Thai, v. 90, n. 3, p. 452-8, 2007.

13. DOLAN, S. E. et al. Increased cardiovascular disease-risk indices in HIV-infected women. J Acquir Immune Defic Syndr, v. 39, n. 1, p. 44-54, 2005.

14. FICHTENBAUM, C. J. et al.; the NIAID AIDS Clinical Trials Group. Pharmacokinetic interactions between protease inhibitors and statins in HIV seronegative volunteers: ACTG Study A5047. AIDS, v. 16, p. 569-77, 2002.

15. FILHO, W. S.; MARTINEZ, T. L. R. Rotina de atendimento em serviço especializado em dislipidemia. In: LOPES, A. C. (Ed.). Dislipidemias da teoria à prática. São Paulo: Ed. Ateneu, 2004. p. 1-19.

16. FONSECA, M. H. G. A.; CARVALHAL, R. C. A. Diabetes e obesidade. In: OLIVEIRA, J. E. P.; MILECH, A. (Ed.). Diabetes mellitus: clínica, diagnóstico, tratamento multidisciplinar. São Paulo: Ed. Atheneu, 2004. p. 299-316.

17. FOULKES, A. S. et al. Associations among race/ethnicity, ApoC-III genotypes, and lipids in HIV-1-infected individuals on antiretroviral therapy. PLoS Med, v. 3, n. 4, p. e52, 2006.

18. GERAIX, J. et al. Bezafibrate for the treatment of hypertriglyceridemia in HIV1-infected patients on highly active antiretroviral therapy. Braz J Infect Dis, v. 10, n. 3, p. 159-64, 2006.

19. GRINSPOON, S.; CARR, A. Cardiovascular risk and body- 
fat abnormalities in HIV-infected adults. N Eng/ J Med, v. 352, n. 1, p. 48-62, 2005.

20. LARANJEIRA, R. R. Tratamento farmacológico do uso nocivo e dependência do álcool. PEC-ABP; 2005.

21. MALITA, F. M. et al. Effects of different types of exercise on body composition and fat distribution in HIV-infected patients: a brief review. Can J Appl Physiol, v. 30, p. 233-45, 2005.

22. MARTÍNEZ, E.; GATELL, J. M. Metabolic abnormalities and body fat redistribution in HIV-1 infected patients: the lipodystrophy syndrome. Curr Opin Infect Dis, v. 12, n. 1, p. 13-19, 1999.

23. MINISTÉRIO DA SAÚDE (MS). Semanas epidemiológicas de 27 a 52, julho a dezembro de 2006, e de 01 a 26, janeiro a junho de 2007. Boletim epidemiológico Aids - ano IV/nº 1. Brasília: Ministério da Saúde, 2007.

24. NOOR, M. A.; LO, J. C. et al. Metabolic effects of indinavir in healthy HIV-seronegative men. AIDS, v. 15, n. 7, p. F11-18, 2001.

25. PERNERSTORFER-SCHOEN, $\mathrm{H}$. et al. Sex differences in HAART-associated dyslipidaemia. AIDS, v. 15, n. 6, p. 725-34, 2001.

26. PETRUCCELLI, J. L. A declaração de cor/raça no censo 2000: estudo preliminar das tabulações avançadas. Rio de Janeiro: Observatório Latino-Americano de Políticas Educacionais (OLPED)/Laboratório de Políticas Públicas da UERJ; 2008. Disponível em: http://lpp-uerj. net/olped/documentos/ppcor/0110.pdf. Acesso em 19 abr. 2008.

27. SALYER, J. et al. Coronary heart disease risks and lifestyle behaviors in persons with HIV infection. J Assoc Nurses AIDS Care, v. 17, n. 3, p. 3-17, 2006.

28. SAMARAS, K. et al. Prevalence of metabolic syndrome in HIV-infected patients receiving highly active antiretroviral therapy using International Diabetes Foundation and Adult Treatment Panel III criteria: associations with insulin resistance, disturbed body fat compartmentalization, elevated C-reactive protein, and [corrected] hypoadiponectinemia. Diabetes Care, v. 30, n. 1, p. 113-19, 2007. Errata em: Diabetes Care, v. 30, n. 2, p. 455, 2007.

29. SHAH, M. et al. The role of diet, exercise and smoking in dyslipidaemia in HIV-infected patients with lipodystrophy. HIV Med, v. 6, n. 4, p. 291-8, 2005.

30. SULLIVAN, A. K.; NELSON, M. R. Are we winning the battle against the human immunodeficiency virus? Br J Hosp Med, v. 58, n. 10, p. 486-8, 1997.

31. SZWARCWALD, C. L. et al. Aids: o mapa ecológico do Brasil, 1982-1994. In: Ministério da Saúde. Coordenação Nacional de DST e Aids. A epidemia da Aids no Brasil: situação e tendências. Brasília: Ministério da Saúde, 1997. p. 27-44.

32. UNAIDS. 2006 report on the global AIDS epidemic. A UNAIDS 10th anniversary special edition. Geneva: UNAIDS, 2006.

33. YUSUF, S. et al.; INTERHEART Study Investigators. Effect of potentially modifiable risk factors associated with myocardial infarction in 52 countries (the INTERHEART study): case-control study. Lancet, v. 364, n. 9438, p. 937-52, 2004. 